\title{
Is there a correlation between infliximab trough levels and the development of adverse events in patients with inflammatory bowel disease?
}

\author{
Eirini Theodoraki ${ }^{1}$, Eleni Orfanoudaki ${ }^{1}$, Kalliopi Foteinogiannopoulou ${ }^{1}$, Evangelia Legaki ${ }^{2}$, Maria Gazouli ${ }^{2}$, \\ Ioannis E. Koutroubakis ${ }^{1}$ \\ ${ }^{I}$ Department of Gastroenterology, University Hospital of Heraklion, Medical School University of Crete, Heraklion; ${ }^{2}$ Department of Basic \\ Medical Sciences, Laboratory of Biology, National and Kapodistrian University of Athens Medical School, Athens, Greece
}

Background/Aims: The measurement of infliximab trough levels (IFX-TLs) in patients with inflammatory bowel disease (IBD) is performed to optimize treatment. However, the association between the development of adverse events (AEs) and IFX-TLs has not been sufficiently studied thus far. To investigate the possible association of IFX-TLs with AEs in Greek patients with IBD receiving maintenance treatment with IFX. Methods: A retrospective analysis of the registry data of the Gastroenterology Department of the University Hospital of Heraklion, from IBD patients with at least one available IFX-TL measurement during the years 2016 to 2017 was conducted. AEs reported 4 months before and 4 months after the measured IFX-TLs were recorded. The IFX-TLs of patients with or without AEs were compared. Results: Of a total of 83 IBD patients (61 Crohn's disease [73\%]; 52 men [63\%]; mean age \pm standard deviation, $43.3 \pm 16.0$ years), 147 measurements of IFX-TLs were available (median $4.69 \mu \mathrm{g} /$ $\mathrm{mL}$ [1.32-9.16]), and 99 AEs (67.3\%, 14 severe) were registered. The median IFX-TL of patients with AEs was $5.79 \mu \mathrm{g} / \mathrm{mL}(1.36-$ 10.25), higher than the median IFX-TL of patients without AEs $(3.40 \mu \mathrm{g} / \mathrm{mL}$ [1.30-5.92]), but the difference was not significant $(P=0.97)$. The presence of infections or dermatologic reactions was not correlated with IFX-TLs. There was no difference in the prevalence of the total AEs ( $66.7 \%$ vs. $73.3 \%, P=0.77)$ or in the analysis of AEs by group between patients with IFX-TLs $\geq 15 \mu \mathrm{g} /$ $\mathrm{mL}$ and patients with IFX-TLs $<15 \mu \mathrm{g} / \mathrm{mL}$. Conclusions: IFX-TLs are not significantly associated with the development of AEs in IBD patients receiving maintenance treatment with IFX. (Intest Res 2021;19:461-467)

Key Words: Adverse drug events; Crohn disease; Infliximab; Infections; Colitis, ulcerative

\section{INTRODUCTION}

In recent decades, the use of biologics in inflammatory bowel disease (IBD) has proven to be an effective choice to achieve patient remission, to reduce the need for corticosteroids and hospitalizations, to avoid surgery and improve patients' quality of life. ${ }^{1,2}$ Anti-tumor necrosis factor (anti-TNF) agents have

Received May 2, 2020. Revised May 25, 2020. Accepted May 25, 2020. Correspondence to loannis E. Koutroubakis, Department of

Gastroenterology, University Hospital of Heraklion, P.O. BOX 1352, 71110 Heraklion, Greece. Tel: +30-2810392254, Fax: +30-2810542085, E-mail:

koutroui@uoc.gr been used in IBD for the last 20 years, and the first approved drug was infliximab (IFX). IFX has been established as an effective therapy from moderate to severe IBD, and it is used both as induction and maintenance treatment. ${ }^{3}$

Anti-TNF agents are widely used in patients with IBD, both for the induction of remission and maintenance treatment. Sometimes it is necessary to use intensified regimens of therapy to achieve remission. Higher IFX trough levels (IFX-TLs) have been associated with higher rates of remission, but we always consider the probability of toxicity of the drugs when dose adjustment is required. ${ }^{4}$ Many adverse events (AEs) have been described. Some of them require further medica- 
tion or even hospitalization and IFX discontinuation. The most common AEs are infections (up to 45\%), with serious infections described in 3\% and opportunistic infections in less than $1 \%$ of patients with IBD receiving IFX. ${ }^{5}$ Dermatological manifestations (up to 40\%), such as psoriasis or psoriasiform lesions and eczemas, infusion reactions (5\%-10\%), systemic lupus, hypersensitivity reactions, cancers and, less frequently, neurological (demyelinating disorders or neuropathies) or musculoskeletal disorders have also been described. ${ }^{5,6}$ Worsening of heart failure and the occurrence of autoimmunity or aplastic anemia have also been associated with the use of the drug. ${ }^{6}$ General symptoms, such as fatigue or dizziness, and abnormal laboratory tests are also often reported. ${ }^{5-7}$

The main therapeutic goal in IBD patients is deep remission, including clinical, biochemical and endoscopic remission. Therapeutic drug monitoring has been suggested as a useful tool for treatment optimization and a helpful way to make therapeutic decisions to achieve the final aim of deep remission. Moreover, monitoring IFX-TLs and antibodies to IFX (ATIs) could be useful for predicting or preventing possible AEs of IFX. ${ }^{8}$ Especially when IFX is used in combination with an immunomodulator, the risk of some AEs may be increased, ${ }^{9}$ and the close monitoring of IFX-TLs could be valuable.

The use of IFX in IBD patients and the development of AEs with IFX treatment have been well described, but data on the association between the AEs of IFX and IFX-TLs or IFX ATIs are limited and rather conflicting. This study aimed to investigate the possible association of IFX-TLs or ATIs with the development of AEs (in total and by AE subgroups) in Greek patients with IBD receiving maintenance treatment with IFX.

\section{METHODS}

\section{Patients}

A retrospective analysis of prospective registry data from consecutive IBD patients receiving maintenance treatment with IFX was conducted. The IBD registry of the University Hospital of Heraklion, where all information and data are available, was used. The study was approved by the Institutional Review Board of University Hospital of Heraklion, Greece (IRB No 9557/13-11-2019) and performed in accordance with the principles of the Declarations of Helsinki. Written informed consents were obtained. An ELISA kit (ELISA; Eagle Biosciences, Nashua, NH, USA) was used for the measurement of IFX-TLs and ATIs in the patients' serum samples. A first measurement was performed in patients who were under mainte- nance treatment with IFX for a median time of 27 months (14-72). After the first measurement of IFX-TLs and ATIs, a second measurement was performed after a 10-month interval in patients who continued treatment. The lower limit of quantitation of IFX-TLs was $0.03 \mu \mathrm{g} / \mathrm{mL}$ and ATIs at $10 \mathrm{UA} /$ $\mathrm{mL}$ was considered as positive. All IBD patients with 1 or 2 available IFX-TL and ATI measurements for the years 2016 to 2017 were included in this study.

A questionnaire about health status, disease activity and any possible adverse reaction that could be correlated with either the disease or the treatment were completed by a physician before every infusion of IFX. Moreover, routine laboratory blood tests obtained at the same time were recorded. Data from all interviews and laboratory tests were retrospectively analyzed by searching for all possible AEs, including infections (respiratory, urinary, and gastrointestinal), dermatological manifestations (psoriasis, psoriasiform lesions, and eczemas), infusion reactions, solid cancers or lymphomas, neurological or musculoskeletal disorders. The time window chosen was the interval between 4 months before and 4 months after each IFX-TL measurement was available. The rationale to choose the specific time window is that these 8 months were closer to each IFX-TLs measurement, so as to increase the possibility these manifestations to be related or not with the levels of IFX. We divided patients into 2 groups according to the presence or absence of AEs. The IFX-TLs and ATIs of patients with or without AEs (total), with or without infections and with or without dermatological manifestations were compared. Moreover, a cutoff point for IFX-TLs of $15 \mu \mathrm{g} / \mathrm{mL}$ was chosen in accordance to previous reports ${ }^{10}$ to examine the safety of IFX in the separate group of the IBD patients who had "extremely" high IFX-TLs.

\section{Statistical Analysis}

Depending on the normality of the data distribution, which was assessed using the Kolmogorov-Smirnov test, mean (standard deviation, $\mathrm{SD}$ ) or median (interquartile range, IQR) values were calculated. Student $t$-test for parametric continuous data and the Mann-Whitney $U$-test for nonparametric continuous data were used for the evaluation of differences among groups. Cox proportional hazards analysis adjusted by the remission time with IFX demonstrating the relationship of the presence of AEs and characteristics of the IBD patients was performed. We considered a difference to be statistically significant if the $P$-value was less than 0.05 . 


\section{RESULTS}

A total of 83 IBD patients receiving maintenance treatment with IFX were included in the study (61 Crohn's disease, 73.5\%; 22 ulcerative colitis, 26.5\%). Among them, 52 patients (63\%) were males, and the mean \pm SD age was $43.3 \pm 16.0$ years. All patients received 5 or $10 \mathrm{mg} / \mathrm{kg}$ IFX per 4, 6, or 8 weeks, and the median (IQR) duration of IFX treatment was 27 months (14-72). Six patients (7.2\%) were receiving intensified doses of IFX at the first measurement, and 17 (20.5\%) were receiving intensified doses of IFX at the second measurement. Eleven patients needed intensification after the first measurement to have better control of their disease. Forty-eight patients (57.8\%) were treated with combination therapy with immunomodulators, either azathioprine or methotrexate (AZA/MTX). The majority of patients had responded to the induction treatment with IFX and maintained in clinical remission under, IFX with or without immunomodulator, as defined by Harvey Bradshaw Index and Simple Clinical Colitis Activity Index scores for Crohn's disease and ulcerative colitis respectively, during the study period. All patients' demographic and clinical data are shown in Table 1.

A total of 147 IFX-TL measurements were available, and the median value was $4.69 \mu \mathrm{g} / \mathrm{mL}$ (1.32-9.16). The presence of AEs (total), recorded 4 months before and 4 months after each IFX-TL measurement, was reported for 99 out of the 147 IFX-TL measurements (67.3\%). In most cases, one single AE was reported, but in 36 out of the 99 measurements (36.3\%), more than one AE was registered. Among all AEs reported, 48 referred to infections ( $48.5 \%$ of all measurements; $75.0 \%$ of respiratory, $12.5 \%$ of urinary tract), and 27 were skin reactions (27.2\% of all measurements); other causes were reported less frequently (Table 2). It should be noted that the skin manifestations reported did not include infusion skin reactions, which were included in the hypersensitivity/infusion reactions.

The median IFX-TLs of patients with AEs (total) were 5.79 $\mu \mathrm{g} / \mathrm{mL}$ (1.36-10.25), which was higher than the median IFXTLs for those without AEs $(3.40 \mu \mathrm{g} / \mathrm{mL}$ [1.30-5.92]), but this difference was not statistically significant $(P=0.97)$. Additionally, patients with infections had higher, but not notably different, IFX-TLs than patients without infections $(5.99 \mu \mathrm{g} / \mathrm{mL}$ [1.64-9.09] vs. $3.75 \mu \mathrm{g} / \mathrm{mL}$ [1.28-9.33], $P=0.16)$. There was also no difference in IFX-TLs regarding the presence or absence of dermatologic reactions $(5.98 \mu \mathrm{g} / \mathrm{mL}[1.26-8.46]$ vs. $4.55 \mu \mathrm{g} / \mathrm{mL}$ [1.34-9.25], $P=0.90$ ) (Fig. 1). Moreover, median ATIs were not different between the patients with or without
Table 1. Demographic and Clinical Characteristics of the 83 IBD Patients Included in the Study

\begin{tabular}{|c|c|}
\hline Characteristics & Value \\
\hline \multicolumn{2}{|l|}{ Sex } \\
\hline Male & $52(63)$ \\
\hline Female & $31(37)$ \\
\hline Age (yr) & $43.3 \pm 16.0$ \\
\hline Disease duration (yr) & $9(6-17)$ \\
\hline \multicolumn{2}{|l|}{ Smoking } \\
\hline Current & $29(35)$ \\
\hline No & $22(27)$ \\
\hline Past & $30(36)$ \\
\hline BMI $\left(\mathrm{kg} / \mathrm{m}^{2}\right)$ & $24.7(20.7-28.1)$ \\
\hline \multicolumn{2}{|l|}{ Disease } \\
\hline$C D$ & $61(73)$ \\
\hline UC & $22(27)$ \\
\hline \multicolumn{2}{|l|}{ Montreal classification (at enrollment) } \\
\hline Ileum (L1 CD) & $23(38)$ \\
\hline Colon (L2 CD) & $13(21)$ \\
\hline lleum + colon (L3 CD) & $25(41)$ \\
\hline Perianal (P CD) & $24(39)$ \\
\hline Inflammatory (B1 CD) & $23(38)$ \\
\hline Stenotic (B2 CD) & $18(30)$ \\
\hline Fistulizing (B3 CD) & $20(32)$ \\
\hline Left-sided colitis (E2 UC) & $8(36)$ \\
\hline Extensive colitis (E3 UC) & $14(64)$ \\
\hline Combination treatment (IMMs) & $48(58)$ \\
\hline AZA & $40(48)$ \\
\hline MTX & $8(10)$ \\
\hline $\mathrm{HBI}$ for $\mathrm{CD}$ & $2(1-3)$ \\
\hline SCCAI for UC & $1(0-3)$ \\
\hline Median time of IFX use at 1st measurement (mo) & $27(14-72)$ \\
\hline Intensified IFX dose at baseline & $6(7)$ \\
\hline $5 \mathrm{mg} / \mathrm{kg} / 6 \mathrm{wk}$ & $2(2)$ \\
\hline $5 \mathrm{mg} / \mathrm{kg} / 4 \mathrm{wk}$ & $1(1)$ \\
\hline $10 \mathrm{mg} / \mathrm{kg} / 8 \mathrm{wk}$ & $3(4)$ \\
\hline Intensified dose after the 1st measurement & $17(21)$ \\
\hline $5 \mathrm{mg} / \mathrm{kg} / 6 \mathrm{wk}$ & $11(13)$ \\
\hline $5 \mathrm{mg} / \mathrm{kg} / 4 \mathrm{wk}$ & $4(5)$ \\
\hline $10 \mathrm{mg} / \mathrm{kg} / 8 \mathrm{wk}$ & $2(2)$ \\
\hline Previous IBD related surgery & $12(15)$ \\
\hline
\end{tabular}

Values are presented as number (\%), mean \pm standard deviation, or median (interquartile range).

IBD, inflammatory bowel disease; BMI, body mass index; $C D$, Crohn's disease; UC, ulcerative colitis; IMMs, immunomodulators; AZA, azathioprine: MTX, methotrexate; HBI, Harvey Bradshaw Index; SCCAI, Simple Clinical Colitis Activity Index; IFX, infliximab. 


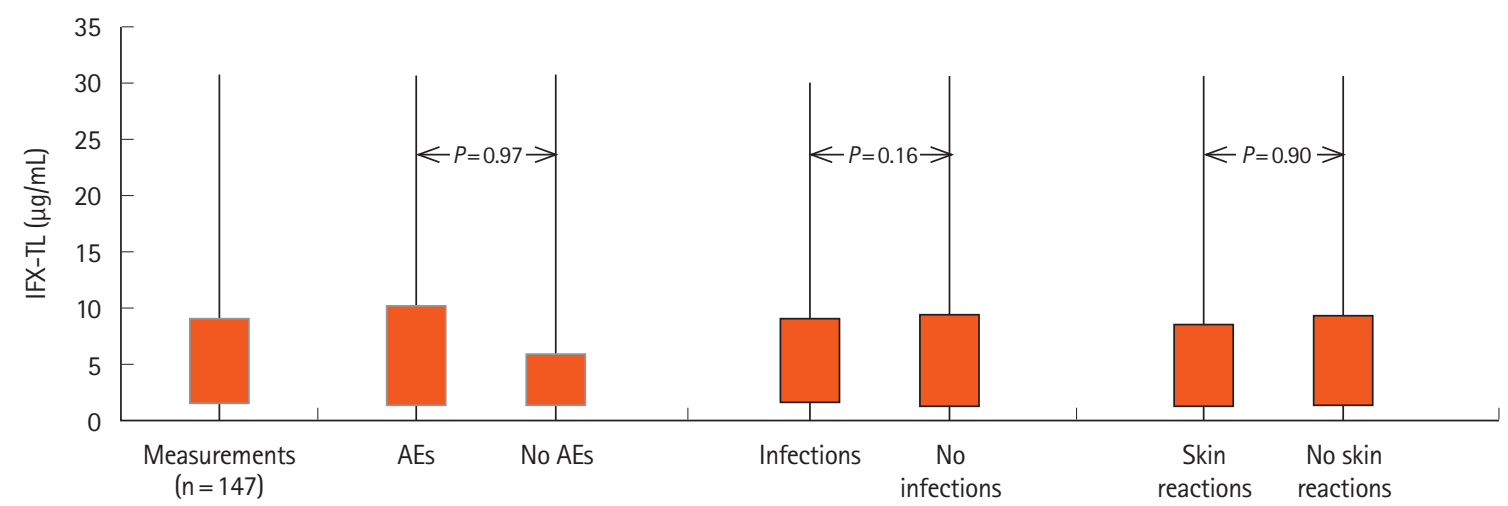

Fig. 1. Median infliximab trough levels (IFX-TLs) of all measurements and comparison of IFX-TLs between patients with and without adverse events (AEs).

Table 2. Frequency of Each Type of AE Reported in All Trough Level Measurements Correlated with AEs $(n=99)$

\begin{tabular}{lc}
\hline Type of AE & No. of patients (\%) \\
\hline Infections & $48(48.5)$ \\
Respiratory & $36(75.0)$ \\
Urinary track & $6(12.5)$ \\
Others & $6(12.5)$ \\
Skin manifestations & $27(27.2)$ \\
Psoriasis or psoriasiform & $8(29.6)$ \\
Skin infections & $9(33.3)$ \\
Others & $10(37.1)$ \\
Hypersensitivity/infusion reactions & $14(14.1)$ \\
Musculoskeletal disorders & $12(12.1)$ \\
Neurological manifestations & $2(2.0)$ \\
Severe AEs & $14(14.1)$ \\
Inferior respiratory infections & $6(6.1)$ \\
CMV infection & $1(1.0)$ \\
Cancers & $5(5.1)$ \\
Extended skin rash & $1(1.0)$ \\
Demyelinating neuropathy & $1(1.0)$ \\
\hline
\end{tabular}

$A E$, adverse event; CMV, cytomegalovirus.

AEs (3.09 UA/mL [2.52-5.63] vs. $2.97 \mathrm{UA} / \mathrm{mL}$ [2.59-4.02], $P=0.19)$.

We used a cutoff of $15 \mu \mathrm{g} / \mathrm{mL}$ to compare the presence of AEs in patients with IFX-TLs. The subgroup with TLs $\geq 15 \mu \mathrm{g} /$ $\mathrm{mL}$ was consisting of the $16 \%$ of all patients and almost $10 \%$ of all IFX-TLs measurements (13 patients, 14 IFX-TLs measurements). The specific cut off was chosen to investigate whether extremely high levels are associated with the presence of any adverse reactions. The comparison of patients with IFX-TLs
Table 3. Cox Proportional Hazards Analysis Adjusted by the Remission Time with IFX Demonstrating the Relationship of the Presence of Adverse Events and Characteristics of the IBD Patients

\begin{tabular}{lcc}
\hline Characteristic & HR $(95 \% \mathrm{Cl})$ & $P$-value \\
\hline Diagnosis (CD) & $1.17(0.63-2.17)$ & 0.62 \\
Age & $0.99(0.97-1.07)$ & 0.31 \\
Female sex & $0.82(0.45-1.48)$ & 0.51 \\
Disease duration & $0.89(0.84-0.93)$ & $<0.001$ \\
Smoking & $1.04(0.73-1.49)$ & 0.82 \\
IFX-TLs & $0.99(0.97-1.03)$ & 0.97 \\
ATIs & $1.05(0.98-1.13)$ & 0.19 \\
Use of immunomodulators & $1.03(0.68-1.57)$ & 0.89 \\
IFX dose intensification & $1.57(0.82-3.02)$ & 0.18 \\
Shorter IFX interval & $1.69(0.61-4.73)$ & 0.35 \\
IBD related surgery & $0.72(0.32-1.59)$ & 0.42 \\
Measurement time & $0.99(0.82-1.22)$ & 0.99 \\
\hline
\end{tabular}

${ }^{a}$ Time between the 2 measurements.

IFX, infliximab; IBD, inflammatory bowel disease; $H R$, hazard ratio; $\mathrm{Cl}$, confidence interval; CD, Crohn's disease; IFX-TLs, IFX trough levels; ATIs, antibodies to IFX.

$\geq 15 \mu \mathrm{g} / \mathrm{mL}$ to those with IFX-TLs $<15 \mu \mathrm{g} / \mathrm{mL}$ showed no significant difference in the prevalence of the total AEs ( $66.7 \% \mathrm{vs}$. $73.3 \%, P=0.77$ ) or in the AE prevalence by group (infections and skin reactions).

Most of the 83 patients (63 patients, $76 \%$ of all patients) had 2 IFX-TL measurements performed within a 10-month interval (126 measurements). The median (IQR) IFX-TL of the 1st measurement (74 available values) was $4.81 \mu \mathrm{g} / \mathrm{mL}$ (1.3912.10), while the median IFX-TL of the 2nd (73 available values) was $4.20 \mu \mathrm{g} / \mathrm{mL}$ (1.10-7.38). Median ATIs (IQR) was 3.44 $\mathrm{UA} / \mathrm{mL}$ (2.92-6.32) and 2.59 UA/mL (2.19-3.03), respectively. AEs were reported in 50 out of 74 (67.5\%) of the 1st measure- 
ments and 49 out of 73 (67.1\%) of the 2nd measurements.

Fourteen patients experienced severe AEs; 6 suffered from inferior respiratory infection, 1 from cytomegalovirus (CMV) systemic infection, 5 from cancer ( 2 colorectal, 1 lymphoma, 1 thyroid, and 1 prostate), 1 from extended skin rash and 1 from demyelinating neuropathy, diagnosed with an electromyography and nerve conduction study and confirmed by a neurologist. All the above reactions required hospitalization and special medication. Regarding the infections (including CMV), patients delayed IFX therapy for 1-2 weeks, but then continued in the same dose. The patient with demyelinating neuropathy and the one with the skin rash changed to other biologic. About cancers, 1 patient died (colorectal cancer), 3 stopped IFX therapy and 1 (prostate cancer) continued IFX in the same dose a while after his surgery. The median IFX-TL in these cases was $7.03 \mu \mathrm{g} / \mathrm{mL}$ (3.21-11.59), which was not significantly different than that in patients without severe AEs (4.32 $\mathrm{\mu g} / \mathrm{mL}$ [1.18-9.15], $P=0.11)$.

In the Cox proportional hazards analysis adjusted by the remission time with IFX, only disease duration was significantly correlated with the development of AEs. No other variable, including sex, age, IBD subtype, smoking status, concomitant immunomodulators, IFX-TLs, ATIs, time between measurements, IFX dose intensification and IBD related surgery were found to be associated with the presence of AEs (Table 3).

\section{DISCUSSION}

Our study showed that higher IFX-TLs in patients with IBD receiving maintenance treatment with IFX were not associated with a higher prevalence of AEs in comparison with similar patients with lower IFX-TLs. Patients with higher IFX-TLs also did not have a higher prevalence of infections or skin manifestations. Even very high IFX-TLs (>15 $\mu \mathrm{g} / \mathrm{mL}$ ) were not found to be toxic for IBD patients.

Although the AEs described above have been associated with the use of IFX, data on the correlation of IFX-TLs with the development of AEs are limited and conflicting. Greener et al. ${ }^{10}$ showed that high IFX-TLs are generally associated with a higher prevalence of AEs, but not by subgroup analysis; the AEs reported were mostly infections and dermatological manifestations. On the other hand, a study by Guiotto et al. ${ }^{11}$ found a significant association ( $P=0.009$; odds ratio, 0.115$)$ between IFX-TLs and the development of AEs. Huang et al. ${ }^{12}$ showed that IFX-TLs were not correlated with the presence of any AE, but low IFX-TLs were associated with infusion reactions. A po- tential role of the persistence of serum ATIs in those patients, followed by low IFX-TLs, could be suggested. Moreover, it has been reported that high IFX-TLs are associated with the presence of dermatological adverse reactions, but the validity of these results and the possible underlying mechanisms remain unclear. $^{12}$ In 2 different studies, Protic et al. ${ }^{13}$ and Cleynen et al. $^{14}$ found no correlation between IFX-TLs and skin adverse reactions. Drobne et al. ${ }^{15}$ primarily studied the rates of infections in patients of different groups regarding IFX-TLs and concluded that there was no difference between the frequencies of infections in the different groups. Coutzac et al., ${ }^{16}$ on the other hand, compared patients with or without paradoxical cutaneous and rheumatological manifestations. They found no significant difference in the IFX-TLs between these groups in their analyses, but it seems that patients with paradoxical rheumatological manifestations (mainly arthralgias) while receiving IFX therapy tended to have lower IFX-TLs.

The results of these studies, including our study, should be supported by larger prospective studies due to the significance of these conclusions. Higher IFX-TLs are frequently needed to achieve clinical and endoscopic remission, and it must be clarified whether higher IFX doses or higher IFX-TLs lead to a higher prevalence of AEs. In our study, we showed that higher IFX-TLs were not correlated with more AEs, infections or dermatological manifestations. A higher dose of the drug does not always mean that higher IFX-TLs will be achieved. This makes the monitoring of IFX-TLs necessary for the optimization of patient therapy and to achieve the final goals of clinical and endoscopic remission and mucosal healing. These results can lead to a safer use of higher doses to achieve higher levels or lessen anti-drug antibodies, meaning a greater probability of remission. ${ }^{17}$

To obtain safer results, we divided our measurements using a high cutoff $(15 \mu \mathrm{g} / \mathrm{mL})$ to study whether higher levels were correlated with more AEs. Further indirect evidence of no correlation of IFX-TLs and AEs is derived from the fact that the reduction of IF-TLs, which appeared over time in the same sample of patients, ${ }^{18}$ was not associated with an equivalent reduction in the presence of AEs. Based on these results, we conclude that high IFX-TLs are not associated with the presence of AEs and remain a safe target for our patients. Unfortunately, the small size of our study group does not permit us to have safe results about the prevalence of some unusual AEs recorded (cancers and neurological manifestations) with the use of IFX or the IFX-TLs.

The use of combined treatment of IFX with AZA/6-mercap- 
topurine or MTX can lessen the probability of autoimmunity and the occurrence of antibodies. As a result, a reduction of infusion reactions can be achieved. In our study, there was no difference between the groups receiving or not receiving combined therapy, which can be explained by the fact that our patients were receiving maintenance IFX therapy, with a median duration of 27 months. Infusion reactions are not expected with the same frequency in patients after the induction period, as our patients were. . $^{19,20}$

In our study, we found a significant correlation between development of AEs and disease duration. This finding could be partially related to the well-known inverse association between disease duration and response to anti-TNF treatment. Moreover, patients with long disease duration are usually older, have longer duration of IFX treatment, have often a history of use of other medication and disease progression to a more complicated phenotype. All the above could be potential risk factors for the development of the observed AEs in our study population.

Among the strengths of our study is that we had 2 measurements of IFX-TLs available for most patients, and the interval between the 2 measurements was 10 months. Furthermore, we searched for AEs close to each measurement, 4 months before and 4 months after, meaning that the AEs recorded did not overlap and were associated with only the specific measurement. Moreover, we studied not only the correlation between IFX-TLs and infections or cancer, which are the most well-described correlations in the literature, but we also included dermatological manifestations and tried to study other AEs as well.

Some limitations of the study should also be noted. The patient sample studied was rather small. Furthermore, this was a retrospective study, so there may have been AEs during the study period that were not mentioned by the patients. Some of the patients who suffered chronically may have underestimated their symptoms or forget to mention some of the previous events. An infection that was rapidly cured or a skin manifestation not observed and mentioned by the patient are some of the events that may have been missed. Another limitation of our study is the relatively short period of 4 months that we chose to collect safety data for our sample. A longer follow-up period would be more acceptable to draw safer conclusions.

In conclusion, in our study, we showed that higher IFX-TLs did not lead to a greater prevalence of AEs. Higher IFX-TLs were not correlated with a higher proportion of infections or skin adverse reactions. Furthermore, this study shows that even very high IFX-TLs did not lead to a higher prevalence of AEs. These conclusions confirm and strengthen other studies with similar published results. This makes the optimization of IFX therapy, including higher target IFX-TLs to achieve clinical remission, endoscopic remission or even mucosal healing, a safe option for IBD patients treated with IFX.

\section{ADDITIONAL INFORMATION}

\section{Funding Source}

The authors received no financial support for the research, authorship, and/or publication of this article.

\section{Conflict of Interest}

No potential conflict of interest relevant to this article was reported.

\section{Author Contribution}

Conceptualization: Koutroubakis IE. Data curation: Theodoraki E. Formal analysis: Theodoraki E, Orfanoudaki E, Foteinogiannopoulou K. Investigation: Theodoraki E. Methodology: Orfanoudaki E, Foteinogiannopoulou K. Project administration: Koutroubakis IE. Writing - original draft: Theodoraki E. Writing - review \& editing: Legaki E, Gazouli M, Koutroubakis IE. Approval of final manuscript: all authors.

\section{ORCID}

Theodoraki E https://orcid.org/0000-0002-8932-0320 Orfanoudaki E https://orcid.org/0000-0002-5426-2757 Foteinogiannopoulou K https://orcid.org/0000-0003-0554-5256 Legaki E

Gazouli M https://orcid.org/0000-0003-4261-2745

Koutroubakis IE https://orcid.org/0000-0002-3295-6811 https://orcid.org/0000-0002-2773-2709

\section{REFERENCES}

1. Schnitzler F, Fidder H, Ferrante M, et al. Mucosal healing predicts long-term outcome of maintenance therapy with infliximab in Crohn's disease. Inflamm Bowel Dis 2009;15:12951301.

2. Colombel JF, Sandborn WJ, Rutgeerts P, et al. Comparison of two adalimumab treatment schedule strategies for moderateto-severe Crohn's disease: results from the CHARM trial. Am J Gastroenterol 2009;104:1170-1179.

3. Papamichael K, Lin S, Moore M, Papaioannou G, Sattler L, Cheifetz AS. Infliximab in inflammatory bowel disease. Ther 
Adv Chronic Dis 2019;10:2040622319838443.

4. van Hoeve K, Dreesen E, Hoffman I, et al. Higher infliximab trough levels are associated with better outcome in paediatric patients with inflammatory bowel disease. J Crohns Colitis 2018;12:1316-1325.

5. Hoentjen F, van Bodegraven AA. Safety of anti-tumor necrosis factor therapy in inflammatory bowel disease. World J Gastroenterol 2009;15:2067-2073.

6. Antoni C, Braun J. Side effects of anti-TNF therapy: current knowledge. Clin Exp Rheumatol 2002;20(6 Suppl 28):S152S157.

7. Lichtenstein L, Ron Y, Kivity S, et al. Infliximab-related infusion reactions: systematic review. J Crohns Colitis 2015;9:806815.

8. Kamperidis N, Middleton P, Tyrrell T, Stasinos I, Arebi N. Impact of therapeutic drug level monitoring on outcomes of patients with Crohn's disease treated with infliximab: real world data from a retrospective single centre cohort study. Frontline Gastroenterol 2019;10:330-336.

9. Quezada SM, McLean LP, Cross RK. Adverse events in IBD therapy: the 2018 update. Expert Rev Gastroenterol Hepatol 2018;12:1183-1191.

10. Greener T, Kabakchiev B, Steinhart AH, Silverberg MS. Higher infliximab levels are not associated with an increase in adverse events in inflammatory bowel disease. Inflamm Bowel Dis 2018;24:1808-1814

11. Guiotto C, Daperno M, Frigerio F, et al. Clinical relevance and inter-test reliability of anti-infliximab antibodies and infliximab trough levels in patients with inflammatory bowel disease. Dig Liver Dis 2016;48:138-143.

12. Huang V, Dhami N, Fedorak D, et al. A study investigating the association of dermatological and infusion reactions to infliximab and infliximab trough levels. Can J Gastroenterol Hepa- tol 2015;29:35-40.

13. Protic M, Münger C, Seibold F. SA1267 psoriasis induced by anti TNF treatment in patients with inflammatory bowel disease: a single center study. Gastroenterology 2014;146(5 Supple 1):S247-S248.

14. Cleynen I, Van Moerkercke W, Billiet T, et al . Anti-TNF-induced skin manifestations in IBD patients: role for increased drug exposure? Gastroenterology 2015;148 (4 Suppl 1):S-108.

15. Drobne D, Kurent T, Golob S, et al. Success and safety of high infliximab trough levels in inflammatory bowel disease. Scand J Gastroenterol 2018;53:940-946.

16. Coutzac C, Chapuis J, Poullenot F, et al. Association between infliximab trough levels and the occurrence of paradoxical manifestations in patients with inflammatory bowel disease: a case-control study. J Crohns Colitis 2015;9:982-987.

17. Moore C, Corbett G, Moss AC. Systematic review and metaanalysis: serum infliximab levels during maintenance therapy and outcomes in inflammatory bowel disease. J Crohns Colitis 2016;10:619-625.

18. Orfanoudaki E, Gazouli M, Foteinogiannopoulou K, et al. Infliximab trough levels are decreasing over time in patients with inflammatory bowel disease on maintenance treatment with infliximab. Eur J Gastroenterol Hepatol 2019;31:187-191.

19. Vermeire S, Noman M, Van Assche G, Baert F, D'Haens G, Rutgeerts P. Effectiveness of concomitant immunosuppressive therapy in suppressing the formation of antibodies to infliximab in Crohn's disease. Gut 2007;56:1226-1231.

20. Ben-Horin S, Waterman M, Kopylov U, et al. Addition of an immunomodulator to infliximab therapy eliminates antidrug antibodies in serum and restores clinical response of patients with inflammatory bowel disease. Clin Gastroenterol Hepatol 2013;11:444-447. 